\title{
Recurrent Ulcerations and Neuropathy in a Filipino Child with Previously Undiagnosed Leprosy: A Case of Lucio Phenomenon in the Philippines
}

\author{
Mia Katrina R . Gervasio, ${ }^{1}$ Aznaida T. Pandapatan ${ }^{1}$ and Belen L. Dofitas ${ }^{2}$ \\ ${ }^{1}$ Section of Dermatology, Department of Medicine, Philippine General Hospital, University of the Philippines Manila \\ ${ }^{2}$ Section of Dermatology, Department of Medicine, College of Medicine and Philippine General Hospital, University of the Philippines Manila
}

\begin{abstract}
Lucio phenomenon is a distinct necrotizing lepra reaction arising from diffuse lepromatous leprosy that may easily be mistaken for other systemic conditions. We report a 17-year old female admitted for a 4-year history of recurrent, extensive ulcerations with crusting and purulent discharge, involving the arms, legs, face, and back. Clinical presentation, slit skin smear and skin biopsy confirmed the diagnosis of Lucio phenomenon and treatment with multidrug therapy resulted in resolution of the ulcerations.
\end{abstract}

Key Words: Lucio phenomenon, leprosy, Hansen's disease

\section{INTRODUCTION}

Lucio phenomenon is a distinct necrotizing lepra reaction arising from diffuse lepromatous leprosy that may easily be mistaken for other systemic conditions. ${ }^{1,2}$ Prompt and accurate diagnosis and treatment remain to be the most important factors in patient management as well as in the reduction of disease burden especially in endemic areas. ${ }^{3,4}$ The severe reaction was previously thought to be exclusive to Mexico but has since been observed in other countries. $^{2}$ Here we report a case of Lucio phenomenon in the Philippines.

\section{CLINICAL CASE}

E-poster presented at the $27^{\text {th }}$ European Academy of Dermatology and Venereology Congress, September 12-16, 2018, Paris, France.

Paper presented at the 2018 PGH Residents' Research Forum, October 9, 2019, 2nd Place, Case Report Category, Manila, Philippines.

E-poster presented at the $41^{\text {st }}$ Annual Convention of the Philippine Dermatological Society, November 7-9, 2018, EDSA Shangri-La, Mandaluyong City, Philippines.

Poster presented at the 2019 American Academy of Dermatology Annual Meeting, March 1-5, 2019, Washington DC, USA.

Corresponding author: Mia Katrina R. Gervasio, MD Section of Dermatology

Department of Medicine

Philippine General Hospital

University of the Philippines Manila

Taft Avenue, Manila 1000, Philippines

Telephone: +632 5548400 local 5105/5106

Email:mrgervasio@up.edu.ph
Our patient is a 17-year-old female from San Jose City, Nueva Ecija who was admitted at the Department of Pediatrics of the Philippine General Hospital for a 4-year history of extensive ulcerations over the bilateral upper and lower extremities, face, and back. She had been intermittently treated with several courses of antibiotics at different hospitals resulting only in the temporary improvement of the lesions.

On admission, she was awake, conversant, bed-bound and in-pain with a visual analog score (VAS) of 7/10. She was not in cardiorespiratory distress and had stable vital signs. She was severely underweight at $30 \mathrm{~kg}$ and a body mass index (BMI) of 13.0. She had pale palpebral conjunctivae and no cervical lymphadenopathies. Chest, lung, heart and abdominal findings were normal. On examination of the skin, she had multiple irregularly-shaped skin-colored and hypopigmented atrophic scars on the forehead and cheeks, 
with some areas of erosion and brown crusting. There was thinning of the outer portion of the eyebrows (madarosis), with no loss of eyelashes. The left ear shows an ill-defined erythematous patch extending from the helix to the lobule. On her arms, legs, back and gluteal area there were multiple well-defined ovoid- and irregularly-shaped erosions and ulcers with an erythematous base and heaped-up brown crusts with areas of purulent discharge. She had thenar and hypothenar atrophy of the bilateral palms as well as hammer toe deformities of the $2^{\text {nd }}$ to $4^{\text {th }}$ digits of both feet. Her scalp, chest, and abdomen were spared (Figure 1).

She had difficulty extending her elbows and knees due to pain. On palpation, there were diffuse nerve enlargement and tenderness of the bilateral radial, ulnar, median, common peroneal and posterior tibial nerves. On motor examination, she had weakness of the bilateral ulnar, median, radial and peroneal nerves. On sensory examination, she had normal blink reflex, intact sensation on the palms, but the soles could not be tested due to wounds.

Complete blood count showed anemia, leukocytosis and an elevated neutrophil count. Serum blood chemistry, renal and hepatic function tests were all within normal limits. Her chest radiograph showed no significant findings. Infectious work-up was done, and wound culture yielded moderate growth of methicillin-resistant Staphylococcus aureus (MRSA). Tissue fungal culture and blood culture also came back negative. Work-up for tuberculosis infection was done, however, sputum and urine acid-fast bacilli smear and tissue tuberculosis polymerase chain reaction $(\mathrm{PCR})$ tests were all negative. Screening for an immunocompromised state was also done and HIV antigen/antibody test, rapid plasma reagin (RPR) test, and hepatitis profile were all non-reactive. To test for leprosy, slit skin smear of 6 sites were done, yielding an average bacterial index of $1+$ with fragmented bacilli thus confirming the diagnosis.

A skin punch biopsy was obtained from the edge of an ulcer on the right leg, and from the edge of a bulla on the right arm. Tissue from both specimens showed moderately dense infiltrates of lymphocytes and foamy histiocytes extending to the subcutaneous tissue. Fite-Faraco stain for leprosy bacilli was done and both sites showed the presence of fragmented bacilli with nerve infiltration, giving a final histologic diagnosis consistent with lepromatous leprosy, in lepra reaction (Figure 2). To determine the etiologic agent, formalin-fixed and paraffin-embedded tissue samples were sent to the University of Texas, MD Anderson Cancer Center for sequencing. Mycobacterium leprae was confirmed to be present in the tissue samples.

Based on the clinical presentation of extensive cutaneous ulcerations, neuritis, and hammer toe deformities, along with a positive slit skin smear and a consistent histopathologic finding, the patient was diagnosed as a case of diffuse lepromatous leprosy with Lucio phenomenon, secondary bacterial infection, anemia of chronic disease and protein-energy malnutrition.
The patient was promptly started on a multi-drug therapy (MDT) regimen of rifampicin, clarithromycyin, and clofazimine. Dapsone was withheld due to the patient's anemia. For the neuritis and lepra reaction, she was started on prednisone and pentoxifylline. Daily bedside debridement of the ulcerations was also done. For the secondary bacterial infection, she was given clindamycin and amikacin. She received a blood transfusion for the anemia and was placed on an up-building diet. To help repair nerve damage, she was started on Vitamin B complex and to increase anti-oxidants, she was started on ascorbic acid and zinc supplementation. Calcium with vitamin D was also given to prevent steroid-induced osteoporosis.

One week after admission, she was discharged improved, with cutaneous inflammation and nerve pain drastically reduced. On follow-up three weeks after discharge, all her ulcers have healed with significant scarring and she had a limitation on the active extension of the left elbow due to contractures (Figure 1). She was wheelchair bound, and still needed assistance when ambulating. She will be given two years of MDT treatment.

\section{DISCUSSION}

Lucio and Alvarado first described Lucio phenomenon in 1852 as a necrotizing lepra reaction occurring as a complication of the non-nodular form of leprosy in Mexico. ${ }^{5}$ In 1948, Latapi and Zamora recognized this reaction histopathologically as a form of vasculitis and emphasized its exclusive occurrence in previously untreated diffuse nonnodular leprosy which they called Lucio's leprosy or pure and primitive diffuse lepromatosis. They termed the distinct lepra reaction as erythema necroticans or Lucio phenomenon. ${ }^{6,7}$

In diffuse non-nodular leprosy, the initial skin involvement is subtle and barely visible. It is clinically characterized by a diffuse infiltration giving an indurated or edematous appearance to the face, hands, and feet. The ears appear erythematous and swollen, and most patients gradually develop alopecia of the eyelashes, eyebrows and body hair. Because of the nonspecific signs and absence of nodules, it is commonly ignored by patients or missed by their clinicians. Thus, the diagnosis is usually made retrospectively, after the manifestation of Lucio phenomenon. This is substantiated in a study conducted by Rea and Jerskey ${ }^{8}$ wherein only four in a series of 30 patients were diagnosed with leprosy prior to the development of Lucio phenomenon. ${ }^{7,1}$

Lucio phenomenon appears approximately one to three years after the initial manifestation of the disease. ${ }^{2}$ The lesions usually begin as crops of painful erythematous or purpuric patches of various sizes with ovoid or angular borders. These evolve into hemorrhagic infarcts with central necrosis that appears as a small blister. It then forms a dark red eschar that falls off a few days later, leaving a pearly white atrophic scar. In other cases, the erythematous patches may 

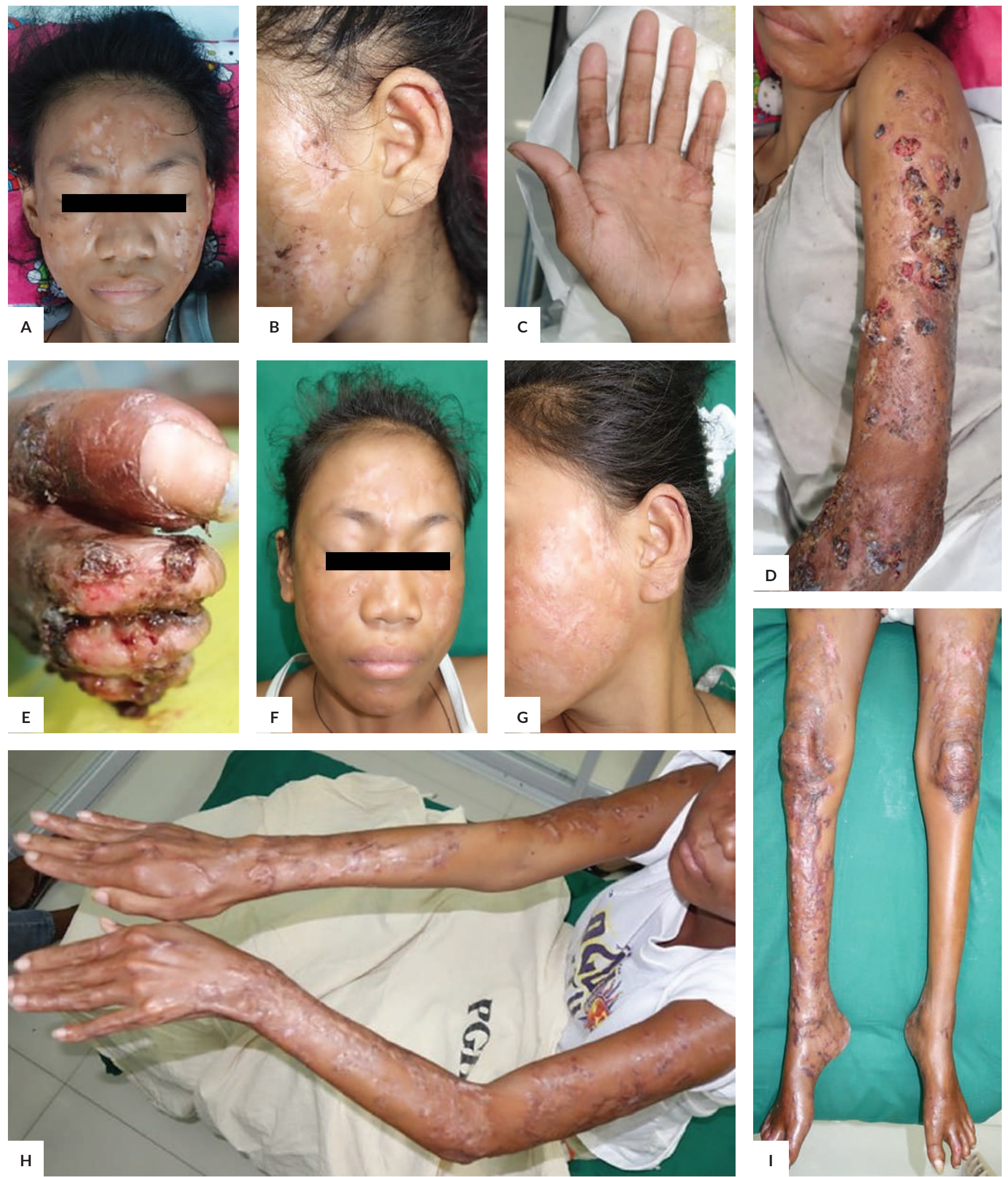

Figure 1. A. Multiple irregularly-shaped hypopigmented atrophic scars on the forehead and cheeks, lateral eyebrow thinning; B. Left ear shows ill-defined erythema and induration; C. Thenar and hypothenar atrophy on the palms; D. Multiple ovoid and irregularly-shaped erosions and ulcerations with thick crusting; E. Hammer toe deformities with ulcerations and crusting, 3 weeks after discharge; $\mathbf{F}$ to $\mathbf{~}$. Complete resolution of ulcerations with atrophic and hypertrophic scarring four weeks after initiation of MDT, prednisone and pentoxifylline. 

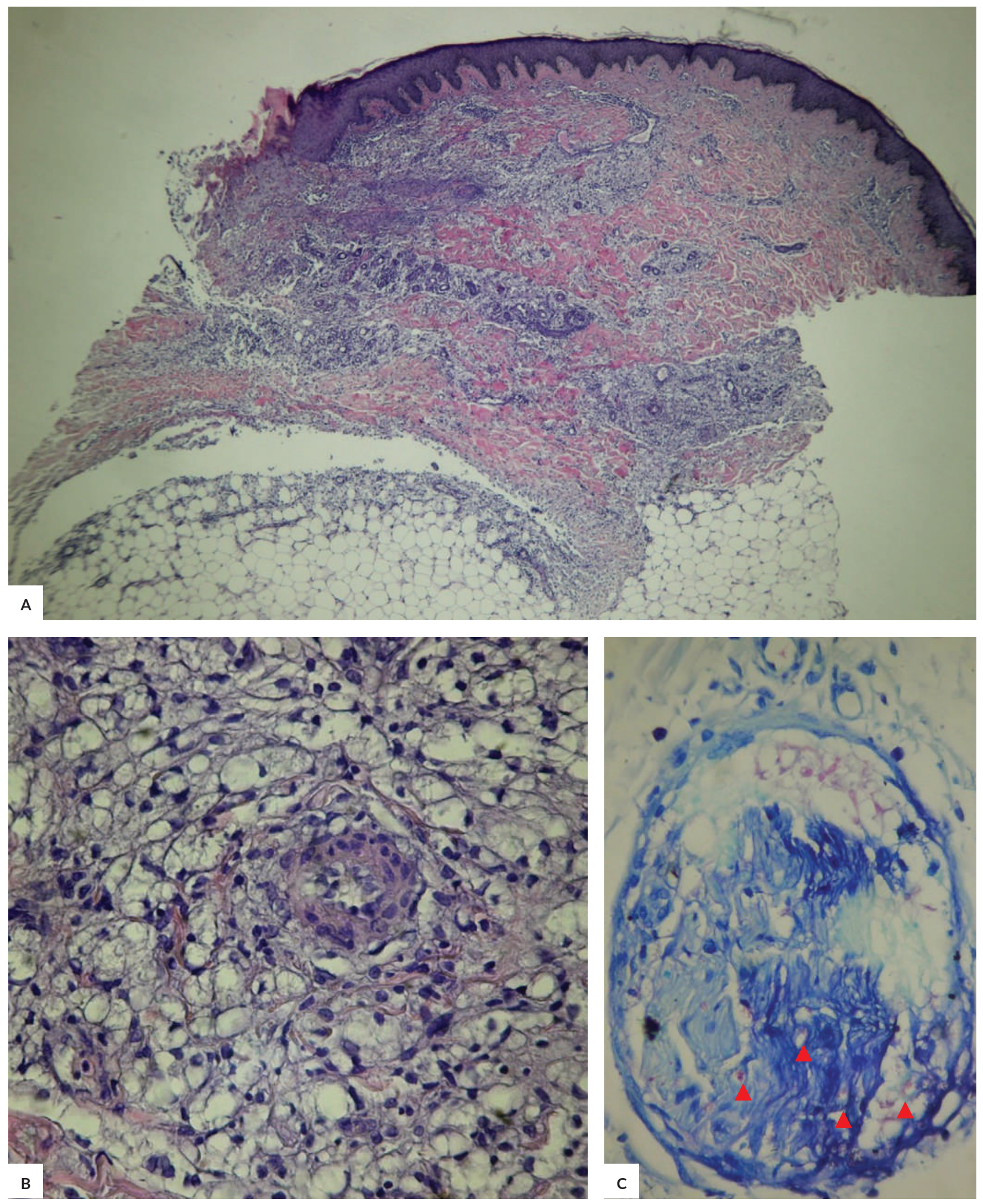

Figure 2. A. Biopsy showed moderately dense infiltrates of lymphocytes and foamy histiocytes extending to the subcutaneous tissue. H\&E, Left leg, Scanning view; B. Foamy histiocytes in the dermis. H\&E, Left leg, 40x; C. Fite faraco stain showing fragmented bacilli with nerve infiltration (arrows), Left leg, 40x. 
form flaccid blisters commonly found in the lower legs. In advance stages, these may become ulcerated and generalize to involve the feet thighs, hands, arms, and less commonly, the trunk and face. The ulcerations may become secondarily infected with bacteria leading to sepsis, a common cause of mortality in such cases. ${ }^{1,2,7,9}$ Other findings such as fever, arthritis, lymphadenopathies, and hepatosplenomegaly may accompany Lucio phenomenon, as well as laboratory findings of anemia, hypoalbuminemia, hypocalcemia, and an elevated erythrocyte sedimentation rate (ESR). Other cases have also shown a false-positive syphilis serology, positive rheumatoid factor, and positive antinuclear factor., ${ }^{2,7,6,10}$

While leprosy is endemic to the Philippines, the diagnosis of leprosy was not readily apparent in this case due to her unrecognized presentation. The erythema and induration of her left ear, as well as lateral eyebrow thinning were subtle signs that could easily be missed. She had no saddle nose deformity or loss of eyelashes that are commonly encountered in advanced cases of the disease. In addition, her hammer toe deformities were masked by extensive ulcerations and crusting on her feet. The most important clinical finding that supported a diagnosis of leprosy in this case was the presence of peripheral neuropathy (nerve tenderness and enlargement) that can be readily differentiated from arthritis on palpation. A slit skin smear was then immediately obtained and confirmed our initial assessment.

Histologically, a review by Costa $^{2}$ in 2005 reported the most commonly observed pathologic changes. These include endothelial cell colonization by bacilli, ischemic epidermal necrosis, necrotizing vasculitis in the superficial dermis, endothelial cell proliferation of vessels in the middermis, and neutrophilic infiltration with neutrophilic nuclear dust. Vargas-Ocampo also studied 30 cutaneous biopsies of Lucio's phenomenon and reported two histologic patterns: the first is leukocytoclastic vasculitis, though to be an immune-mediated process towards the bacilli, while the second is a mild mononuclear infiltrate accompanied by the proliferation of endothelial cells, vascular thrombosis, and necrosis. ${ }^{11}$ These histologic changes underpin the proposed mechanism by which Lucio phenomenon is thought to occur: Patients exhibit an exceptionally deficient immune response, allowing free replication of the Mycobacterium in endothelial cells directly causing vascular thrombosis and necrosis, or indirectly increasing the exposure of mycobacterial antigen to antibodies leading to vasculitis and infarction.,11

The two skin biopsies we obtained from this patient, while consistent with leprosy and an ongoing lepra reaction, were not specific to Lucio phenomenon. In fact, the findings of lymphocytic and foamy histiocytic infiltrates extending to the subcutaneous tissue may also be present in a type II lepra reaction (ENL). Despite using a $6 \mathrm{~mm}$ punch as recommended by Rea and Jerskey, ${ }^{8,12}$ there was no evidence of endothelial cell infiltration, endothelial cell proliferation or vascular necrosis. Although, we did find evidence of leukocytoclastic vasculitis in subsequent biopsies obtained after discharge. In any case, there is no consensus with regards to the underlying histopathological abnormality in Lucio phenomenon, and factors such as sampling site and stage of evolution of the lesion could influence the histologic appearance. On the basis of a consistent clinical picture and characteristic evolution of lesions, a diagnosis of Lucio phenomenon can still be made for this patient.

Following the recommendations of the World Health Organization, MDT remains to be the cornerstone of the management of any form of leprosy, and its prompt initiation is associated with a better prognosis. ${ }^{2,4}$ Beyond the initiation of MDT, there is currently no consensus regarding the specific treatment of Lucio phenomenon as it is a relatively rare condition. Thus, treatment is generally empiric and based on case reports of successfully treated cases. ${ }^{2}$ Some have reported successful treatment solely through MDT, ${ }^{7}$ whereas others have included oral corticosteroids, thalidomide, clofazimine or pentoxifylline in varying combinations, similar to the management of other lepra reactions. For this particular case, we included prednisone to manage the neuritis and opted to give pentoxifylline, a xanthine derivative that improves peripheral blood flow and enhances peripheral tissue oxygenation. Pentoxifylline poses lesser risk compared to thalidomide ${ }^{13}$ and acts more rapidly compared to clofazimine. ${ }^{13,14}$ Clinically, our patient's response to the treatment has been rapid, with an immediate decrease in pain upon initiation of oral corticosteroids and complete healing of her ulcerations after three weeks.

\section{Acknowledgments}

We would like to thank Dr. Xiang Han, Dr. Johanna Lazo-Dizon and Dr. Patricia Asuncion for their invaluable contributions to the management of this case.

\section{Disclaimer}

The views expressed in this article are the authors own and do not reflect the views of the institution.

\section{Statement of Authorship}

All authors approved the final version submitted.

\section{Author Disclosure}

All authors declared no conflict of interest.

\section{Funding Source}

This paper was funded by the authors. No external funding agency.

\section{REFERENCES}

1. Jurado F, Rodriguez O, Novales J, Navarrete G, Rodriguez M. Lucio's leprosy: A clinical and therapeutic challenge. Clin Dermatol [Internet]. 2015;33(1):66-78. Available from: http://dx.doi.org/10.1016/j. clindermatol.2014.07.004 
2. Costa IMC, Kawano LB, Pereira CP, Nogueira LSC. Lucio's phenomenon: a case report and review of the literature. Int J Dermatol [Internet]. 2005 Jul 1 [cited 2017 Sep 19];44(7):566-71. Available from: http://doi.wiley.com/10.1111/j.1365-4632.2005.02566.x

3. Walker SL, Lockwood DNJ. The clinical and immunological features of leprosy. Br Med Bull. 2006;77-78(1):103-21.

4. World Health Organization. Expert Committee on Leprosy. Eigth Report. World Health Organ Tech Rep Ser. 2012;(968):1-61.

5. Arnold HL. Diffuse Lepromatous Leprosy of Mexico (Spotted Leprosy of Lucio). Arch Derm Syphilol [Internet]. 1950 Apr 1 [cited 2017 Sep 22];61(4):663. Available from: http://archderm.jamanetwork.com/ article.aspx?doi=10.1001/archderm.1950.01530110129010

6. Ang P, Tay YK, Ng SK, Seow CS. Fatal Lucio's phenomenon in 2 patients with previously undiagnosed leprosy. J Am Acad Dermatol. 2003;48(6):958-61.

7. Choon SE, Tey KE. Lucio's phenomenon: A report of three cases seen in Johor, Malaysia. Int J Dermatol. 2009;48(9):984-8.

8. Rea TH, Jerskey RS. Clinical and histologic variations among thirty patients with Lucio's phenomenon and pure and primitive diffuse lepromatosis (Latapi's lepromatosis). Int J Lepr Other Mycobact Dis [Internet]. 2005 Sep [cited 2017 Sep 22];73(3):169-88. Available from: http://www.ncbi.nlm.nih.gov/pubmed/16830639
9. Han XY, Seo YH, Sizer KC, Schoberle T, May GS, Spencer JS, et al. A new Mycobacterium species causing diffuse lepromatous leprosy. Am J Clin Pathol. 2008;130(6):856-64.

10. Guedes-barbosa LS, Batista E V., Martins DC, Neder L, Crepaldi N, Martins E V. Necrotizing Cutaneous Vasculitis in Multibacillary Leprosy Disease (lucio'. Jcr J Clin Rheumatol [Internet]. 2008 Feb 1 [cited 2017 Sep 18];14(1):57-9. Available from: https://insights.ovid. com/pubmed?pmid $=18431106$

11. Vargas-Ocampo F. Diffuse leprosy of Lucio and Latapí: A Histologic study. Lepr Rev [Internet]. 2007;78(3):248-60. Available from: http:// www.ncbi.nlm.nih.gov/pubmed/18035776

12. Han XY, Jessurun J. Severe leprosy reactions due to Mycobacterium lepromatosis. Am J Med Sci [Internet]. 2013 Jan [cited 2017 Sep 24];345(1):65-9. Available from: http://www.ncbi.nlm.nih.gov/ pubmed/23111393

13. Cuevas J, Rodríguez-Peralto JL, Carrillo R, Contreras F. Erythema Nodosum Leprosum: Reactional Leprosy. Semin Cutan Med Surg. 2007;26(2):126-30.

14. Souza CS, Roselino AM, Figueiredo F, Foss NT. Lucio's phenomenon: clinical and therapeutic aspects. Int J Lprosy other Mycobact Dis Off organ Int Lepr Assoc [Internet]. 2000;68(4):417-25. Available from: http://www.ncbi.nlm.nih.gov/pubmed/11336069 